\title{
Density Prediction Models for Energetic Compounds Merely Using
}

\section{Molecular Topology}

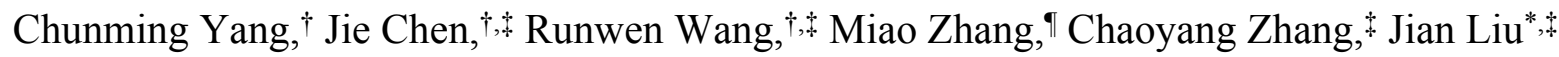

\section{Supporting Information}

Table S1. Molecular features used in current MEGNet model.

\begin{tabular}{ll}
\hline atom feature & description \\
\hline atom type & $\mathrm{C}, \mathrm{H}, \mathrm{O}, \mathrm{N}$ (one-hot) \\
chirality & $\mathrm{R}$ or S(one-hot) \\
is in Ring & whether the atom in ring(one-hot) \\
hybridization & sp, sp2, sp3(one-hot) \\
electron(Donor, Acceptor) & $\begin{array}{l}\text { whether the atom is an electrons acceptor or donor(one-hot) } \\
\text { whether the atom belongs to an aromatic ring(one-hot) }\end{array}$ \\
\hline aromaticity & description \\
\hline bond feature & shortest graph distance between atoms. \\
graph distance matrix & distance valued on Gaussian basis \\
expanded distance & whether the atoms in the bond are in the same ring(one-hot) \\
is in ring & single, double, triple, or aromatic (one-hot) \\
bond type & description \\
\hline global feature & molecular weight divided by the number of atoms(float) \\
\hline average atomic weight & number of bonds divided by number of atoms(float) \\
bonds per atom &
\end{tabular}

Table S2. Adjustments of the main hyperparameters via orthogonal tests with 4 factors and 3 levels.

\begin{tabular}{ccccccc}
\hline BatchSize & NumBlock & OutDim & $\begin{array}{c}\text { DropOu } \\
\text { t }\end{array}$ & MAE & RMSE & $\mathbf{R}^{\mathbf{2}}$ \\
\hline 32 & 1 & 32 & 0.05 & 0.033 & 0.042 & 0.901 \\
32 & 3 & 128 & 0.1 & 0.035 & 0.045 & 0.887 \\
32 & 5 & 64 & 0.2 & 0.028 & 0.035 & 0.930 \\
64 & 1 & 128 & 0.2 & 0.184 & 0.189 & -1.053 \\
64 & 3 & 64 & 0.05 & 0.067 & 0.073 & 0.695 \\
64 & 5 & 32 & 0.1 & 0.031 & 0.045 & 0.886 \\
128 & 1 & 64 & 0.1 & 0.052 & 0.061 & 0.787 \\
128 & 3 & 32 & 0.2 & 0.040 & 0.049 & 0.864 \\
128 & 5 & 128 & 0.05 & 0.129 & 0.134 & -0.022 \\
\hline
\end{tabular}




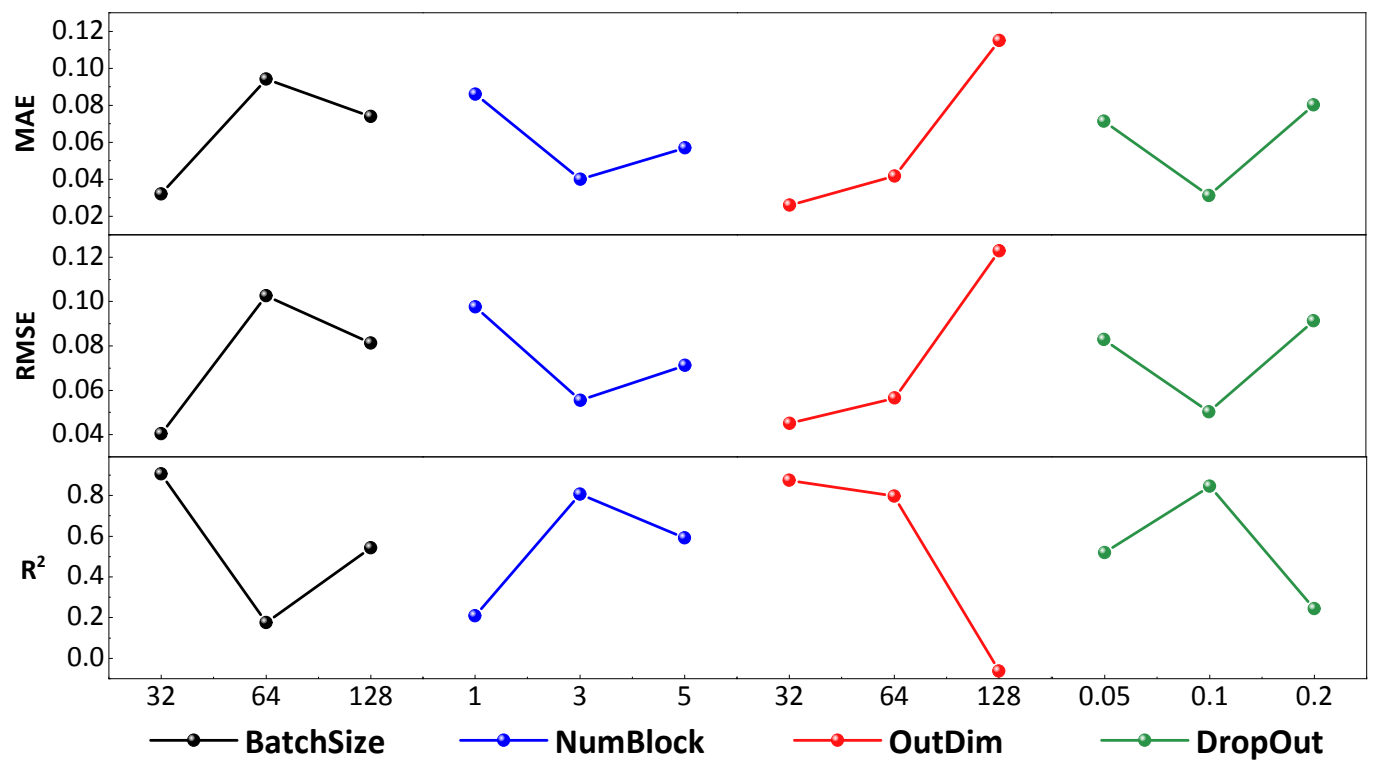

Figure S1. Trend analysis for orthogonal tests. For each of the 4 hyperparameters BatchSize, NumBlock, OutDim and Dropout, the maximum R2 and the minimum MAE and RMSE correspond to the value of $32,3,32$ and 0.1 respectively. The recommended hyperparameters set for current dataset and model is BatchSize $=32$, NumBlock=3, OutDim=32 and Dropout $=0.1$.

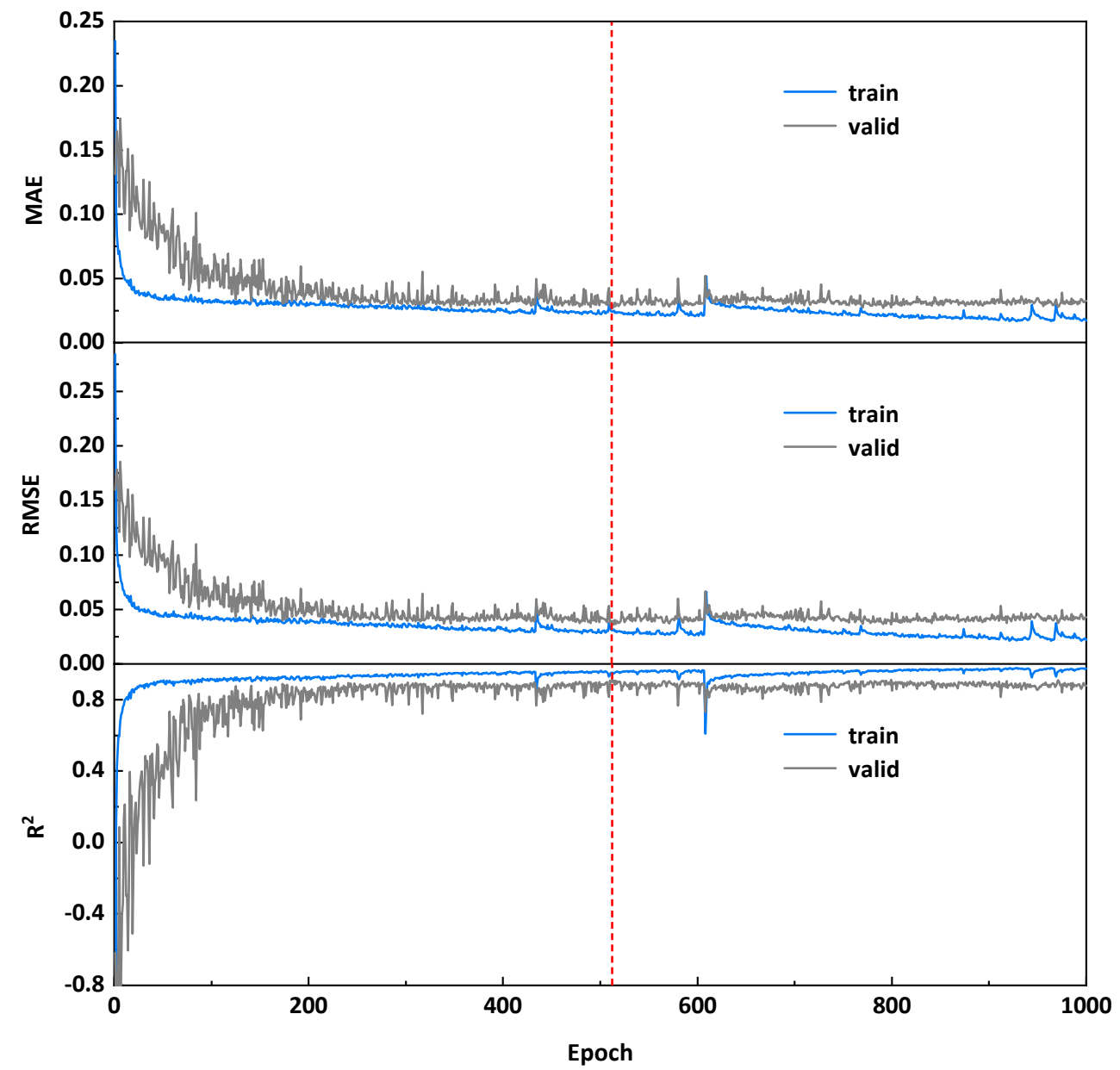

Figure S2. Precision indicators of validation set evolving with training epoch. MAE and RMSE are in $\mathrm{g} / \mathrm{cm}^{3}$. The values of MAE, RMSE and R2 for training set can continue to get better after epoch 512 , but that for validation set became worse, indicating over fitting. 
Table S3 Prediction results from our 4 models and the GAM of Keshavarz by using the test set, unit of density is $\mathrm{g} / \mathrm{cm}^{3}$.

\begin{tabular}{|c|c|c|c|c|c|c|c|c|c|c|c|c|}
\hline CCDC Name & structure & $\rho_{\operatorname{Exp}}$ & $\rho_{G A M}$ & $\begin{array}{l}\rho_{D F T-} \\
O S P R\end{array}$ & $\rho_{\text {svm }}$ & $\rho_{R F}$ & $\rho_{G N N}$ & $\Delta \rho_{G A M}$ & $\begin{array}{l}\Delta \rho_{D F T-} \\
O S P R\end{array}$ & $\Delta \rho_{s v m}$ & $\Delta \rho_{R F}$ & $\Delta \rho_{G N N}$ \\
\hline ATOPOP & & 1.223 & 1.098 & 1.274 & 1.314 & 1.315 & 1.296 & -0.125 & 0.051 & 0.090 & 0.091 & 0.072 \\
\hline BICWEP & $\mathrm{H}_{2}$ & 1.887 & 1.891 & 1.796 & 1.761 & 1.841 & 1.848 & 0.004 & -0.091 & -0.126 & -0.047 & -0.039 \\
\hline BINSAS & & 1.932 & 1.935 & 1.885 & 1.816 & 1.836 & 1.857 & 0.003 & -0.047 & -0.116 & $\begin{array}{c}-0.096 \\
\end{array}$ & -0.075 \\
\hline COWNUX & & 1.303 & 1.137 & 1.274 & 1.309 & 1.275 & 1.257 & -0.166 & -0.028 & 0.006 & -0.028 & -0.045 \\
\hline
\end{tabular}




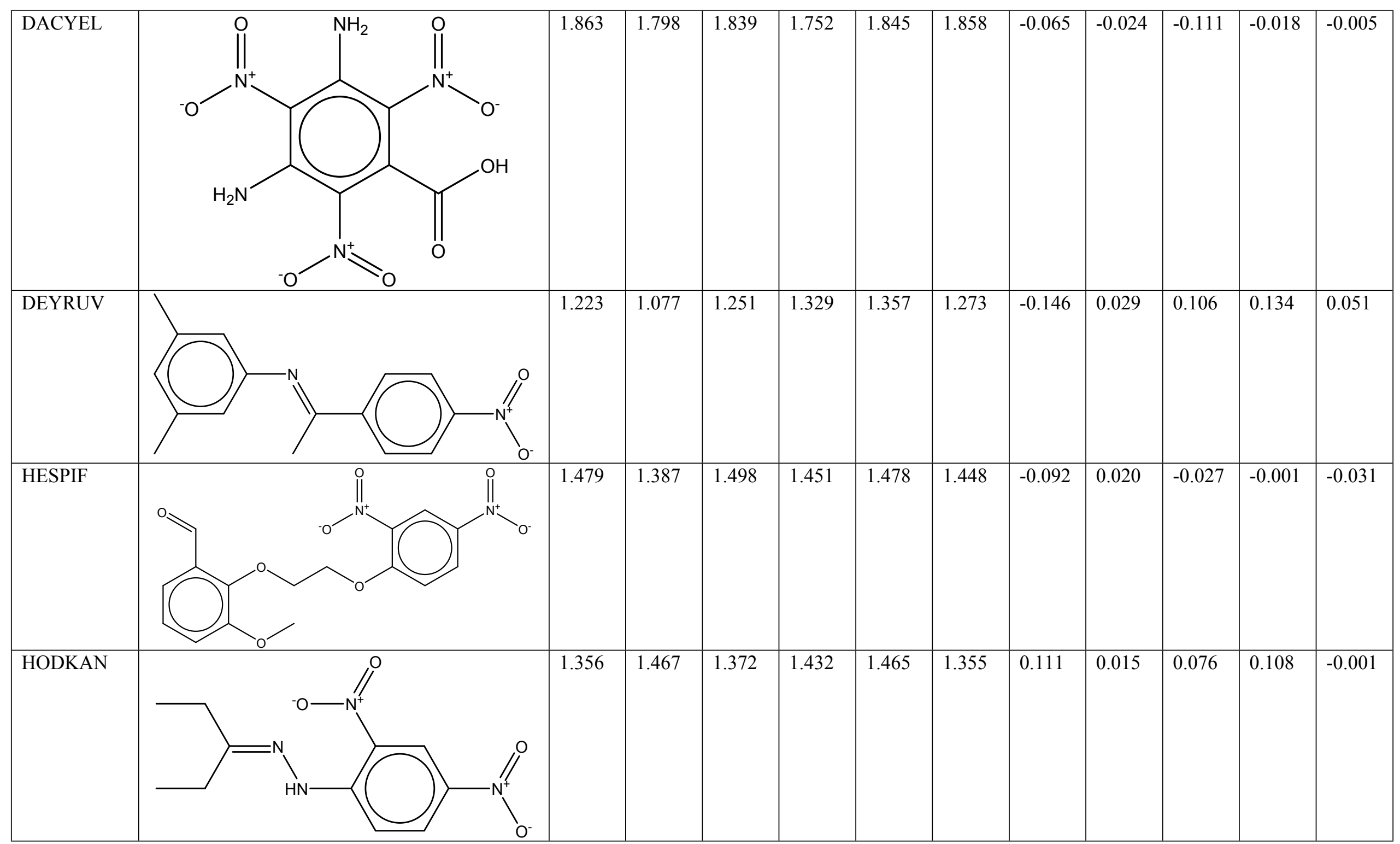




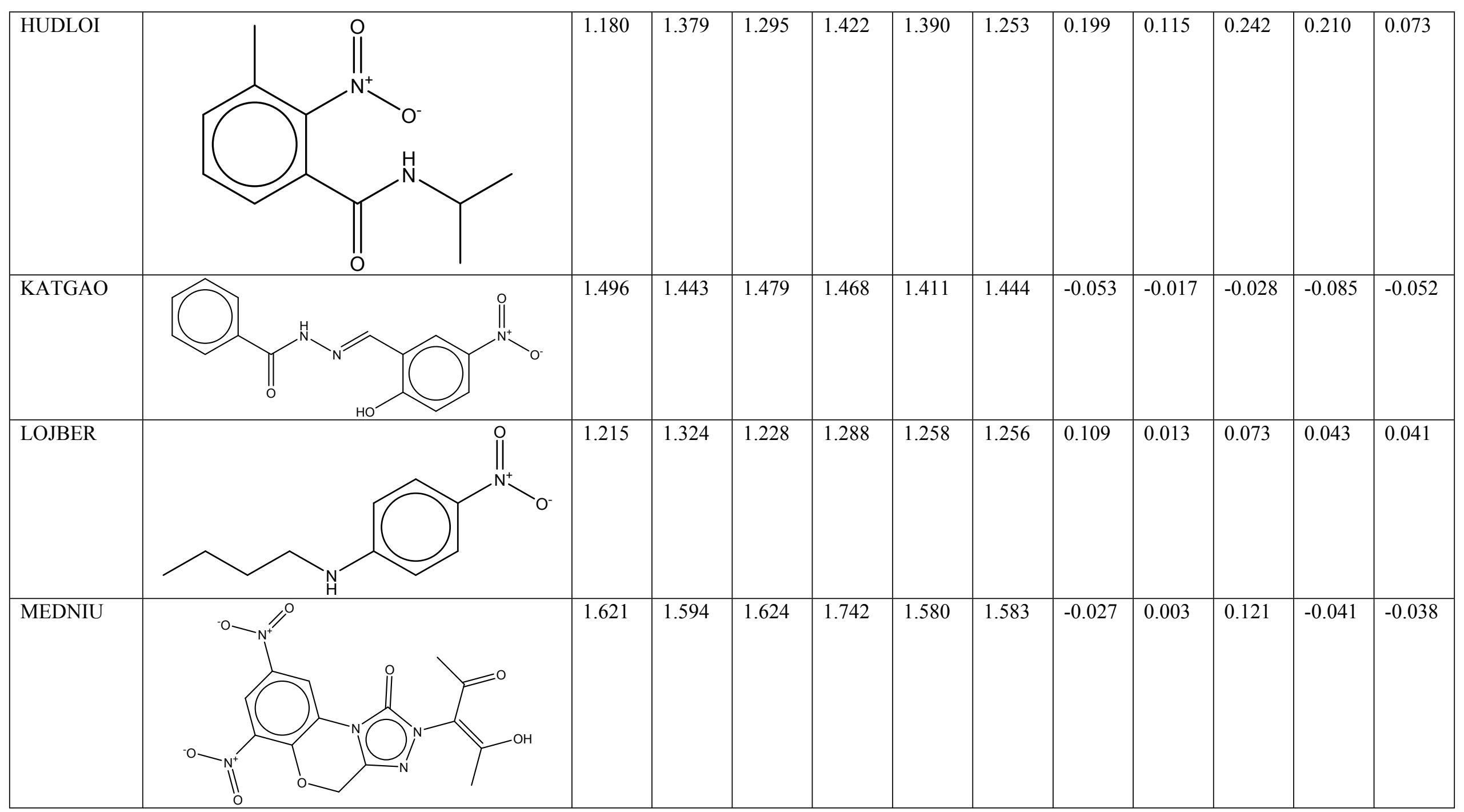




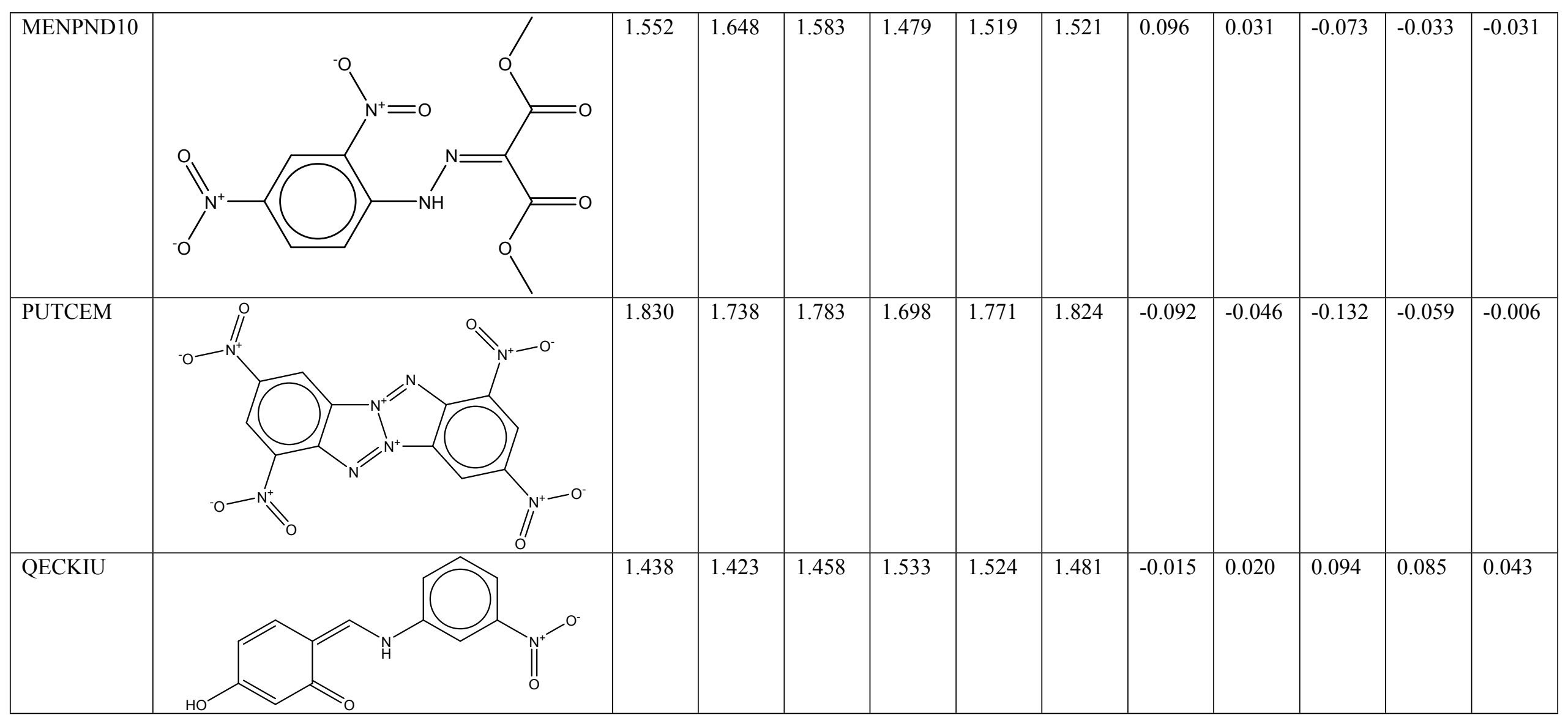




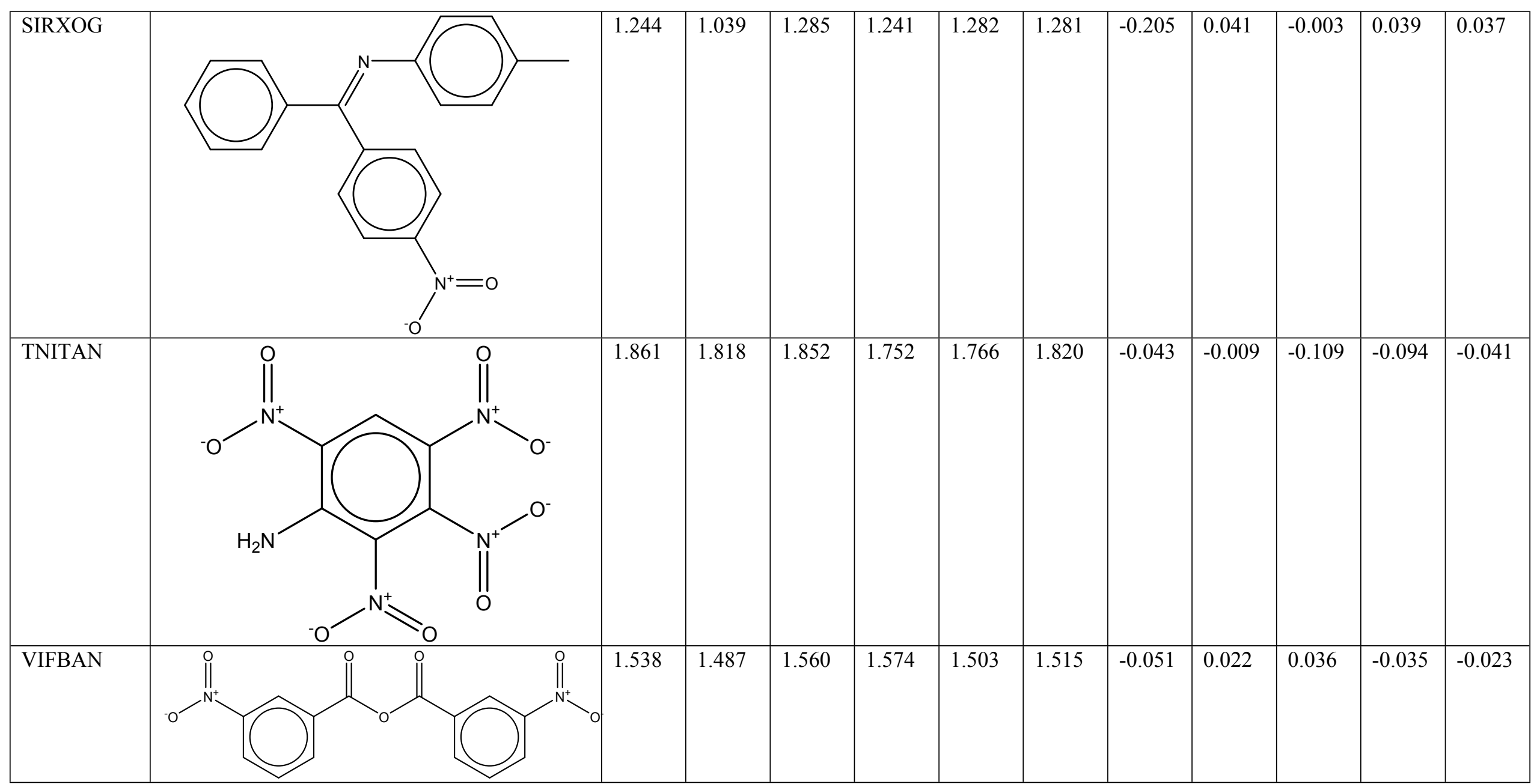




\begin{tabular}{|c|c|c|c|c|c|c|c|c|c|c|c|c|}
\hline ZUCMOB & O & 1.390 & 1.368 & 1.412 & 1.461 & 1.487 & 1.400 & -0.022 & 0.022 & 0.072 & 0.098 & 0.010 \\
\hline AZAXEG & / & 1.642 & 1.730 & 1.623 & 1.581 & 1.459 & 1.619 & 0.088 & -0.018 & -0.061 & -0.183 & -0.022 \\
\hline CIVVEI10 & & 1.688 & 1.590 & 1.682 & 1.613 & 1.583 & 1.722 & -0.098 & -0.006 & -0.075 & -0.106 & 0.034 \\
\hline
\end{tabular}




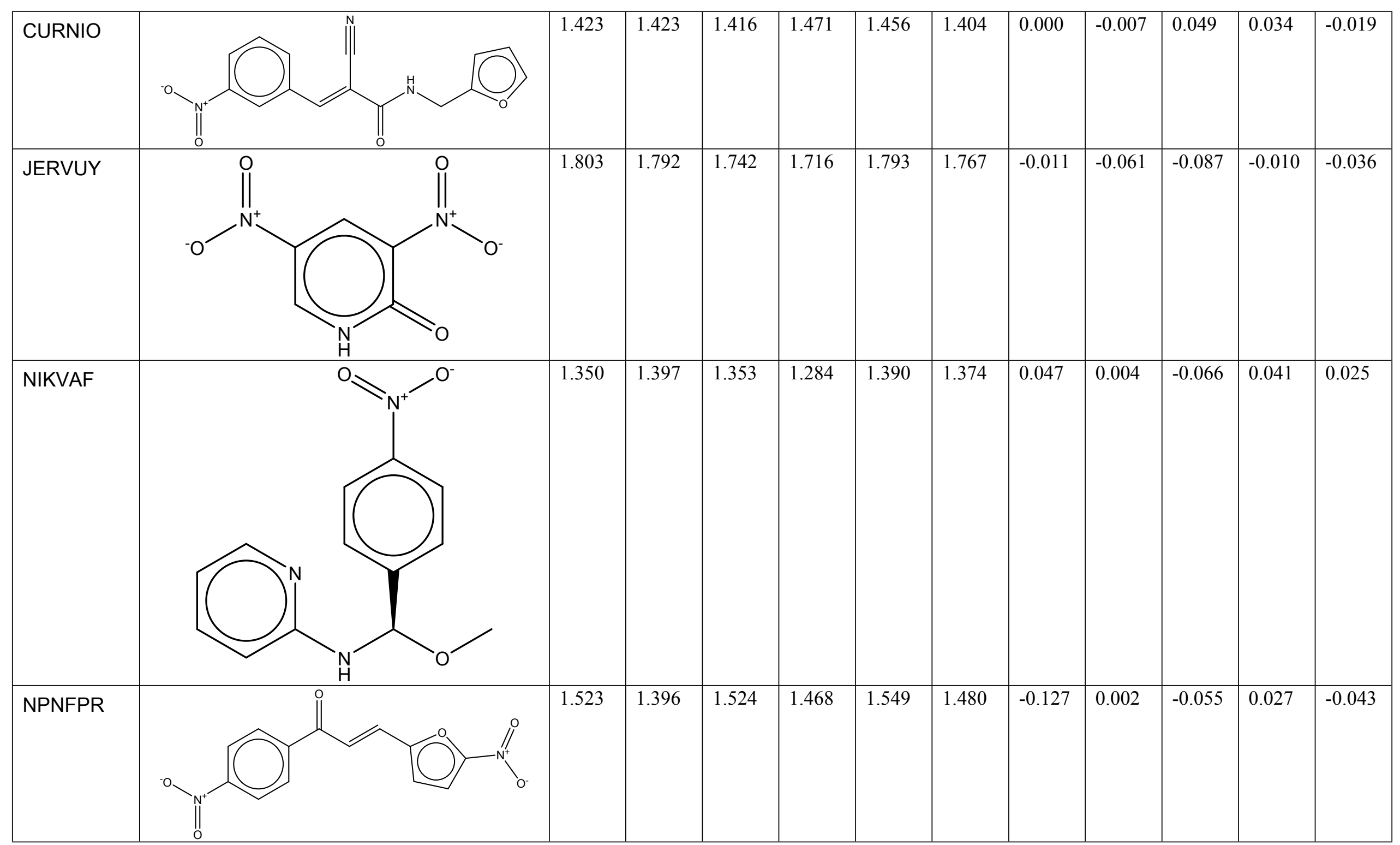




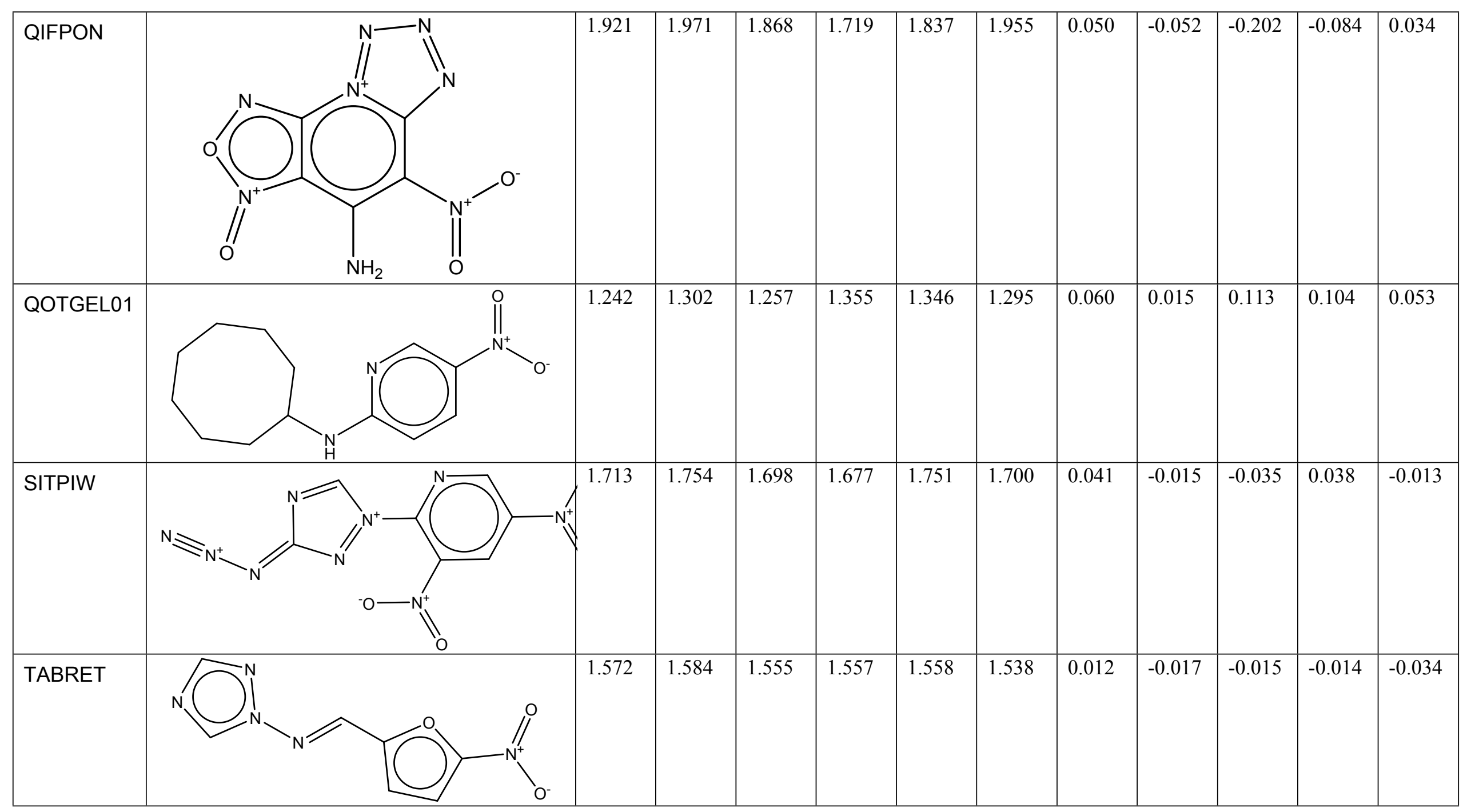




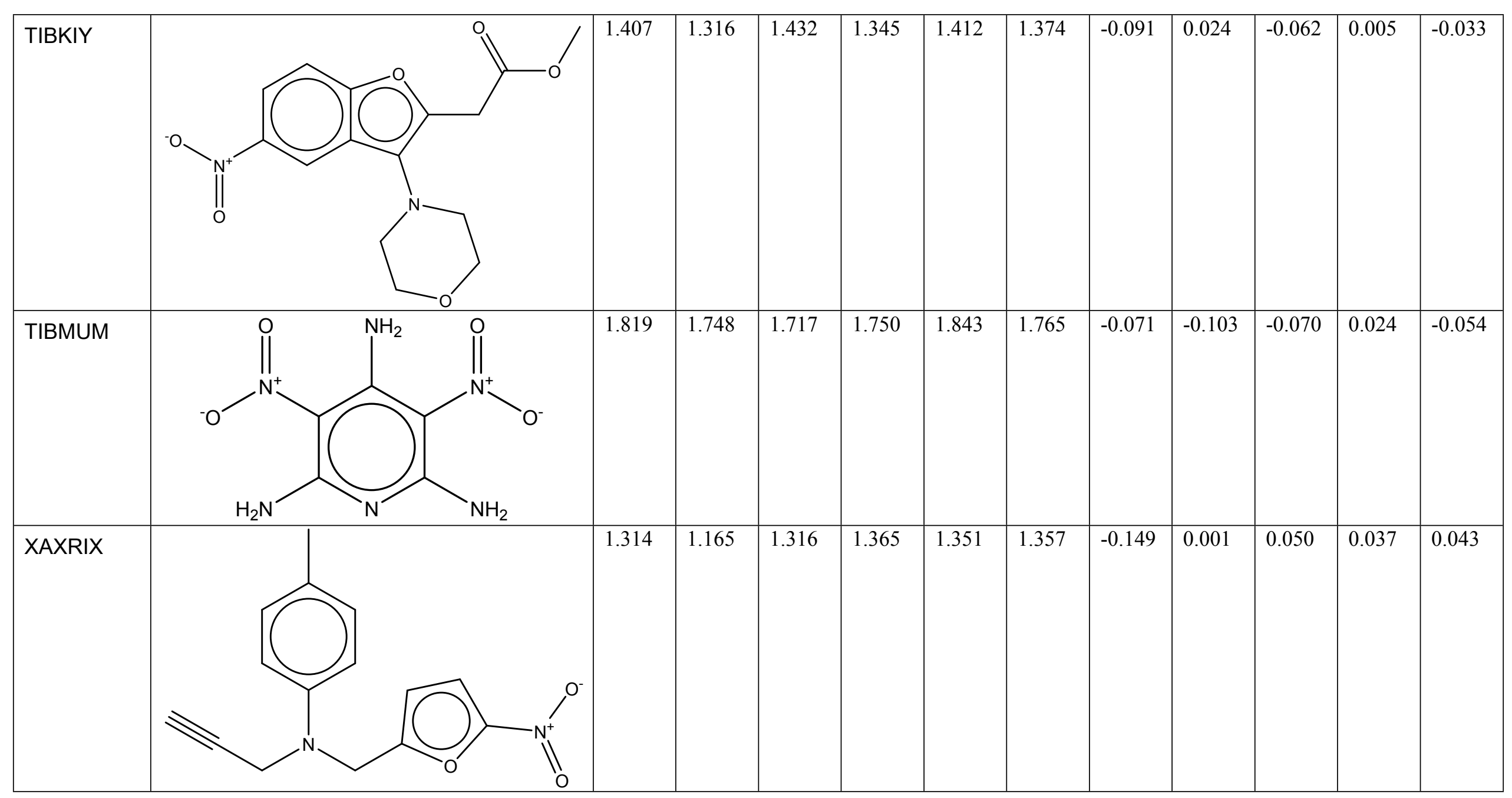




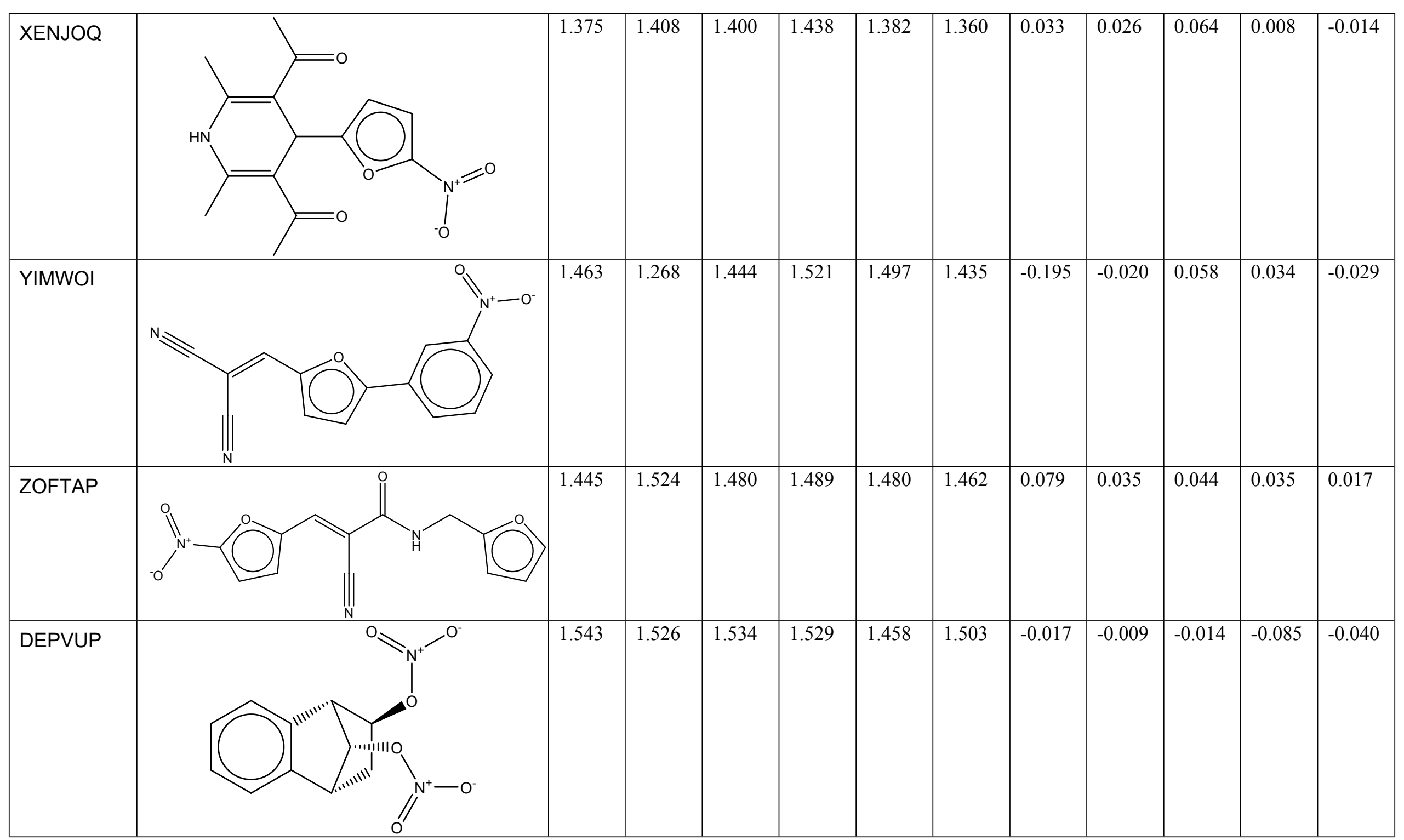




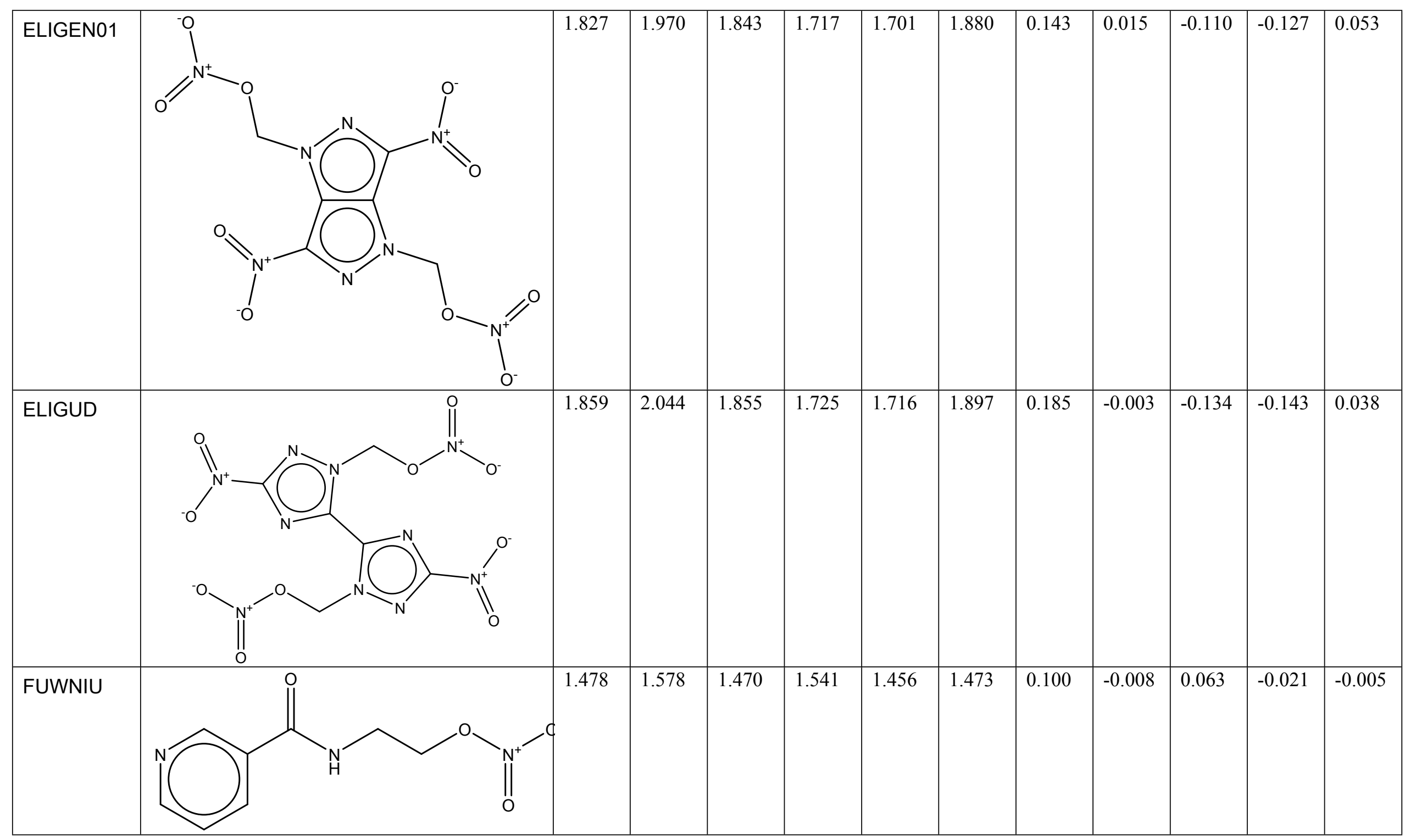




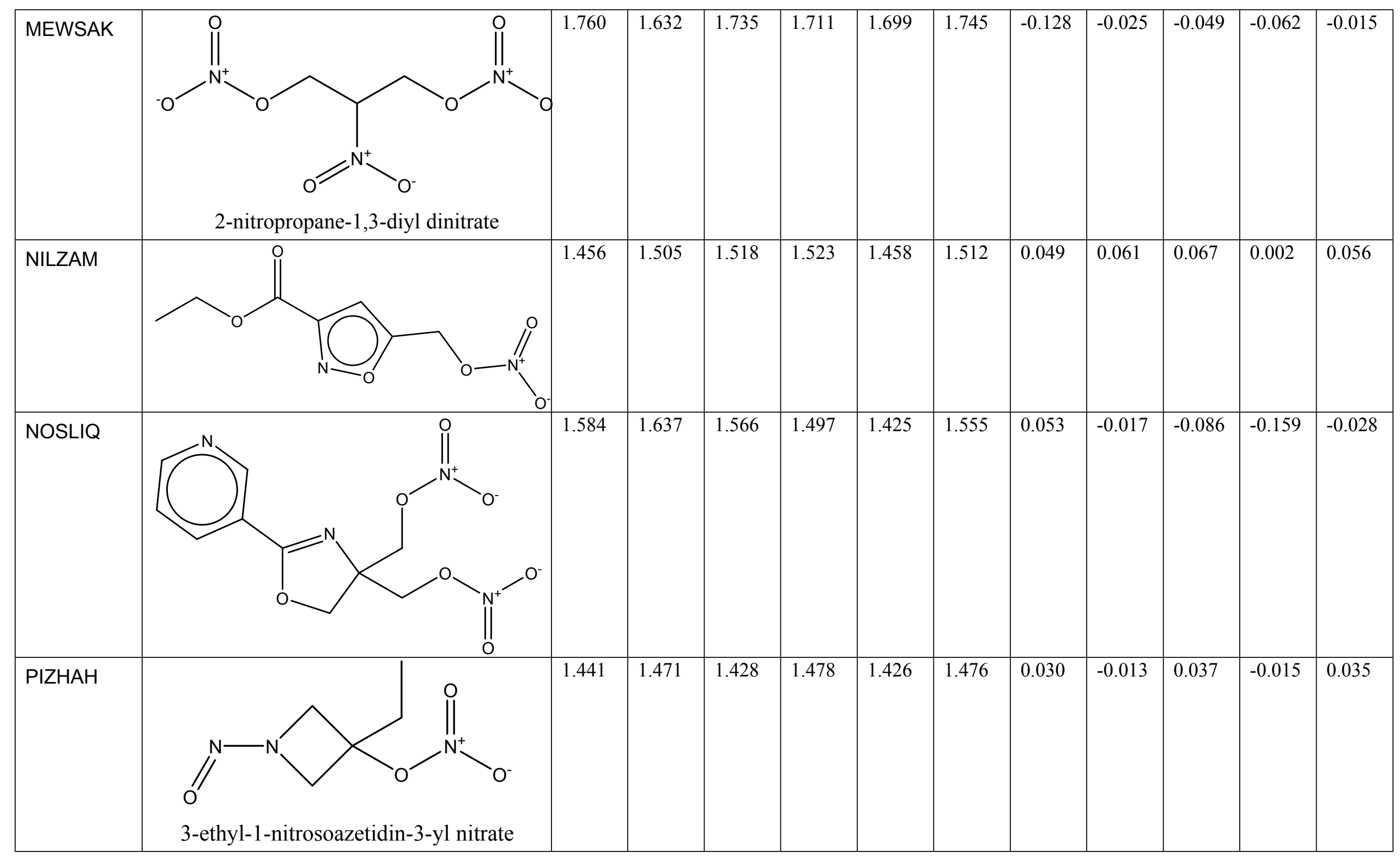




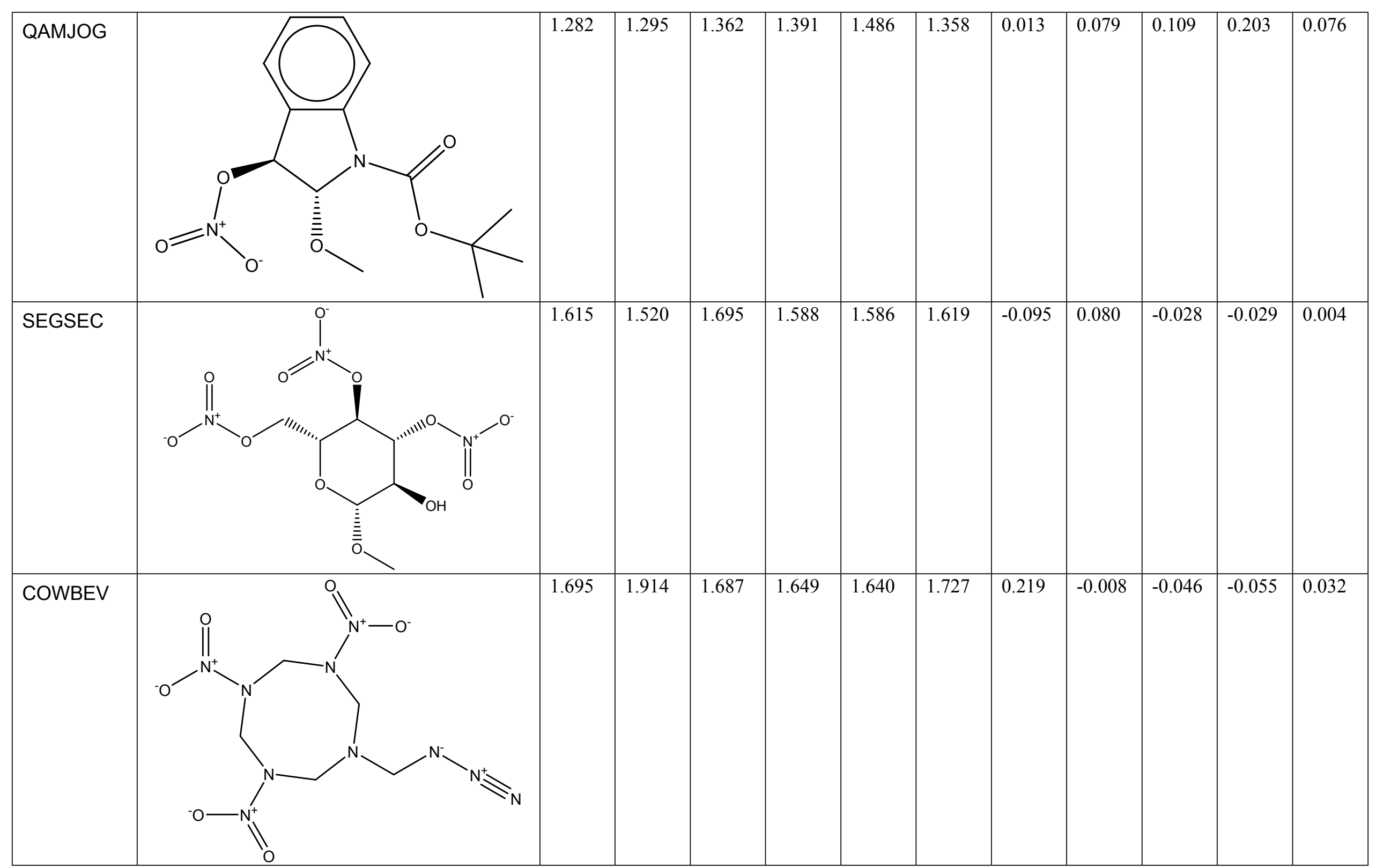




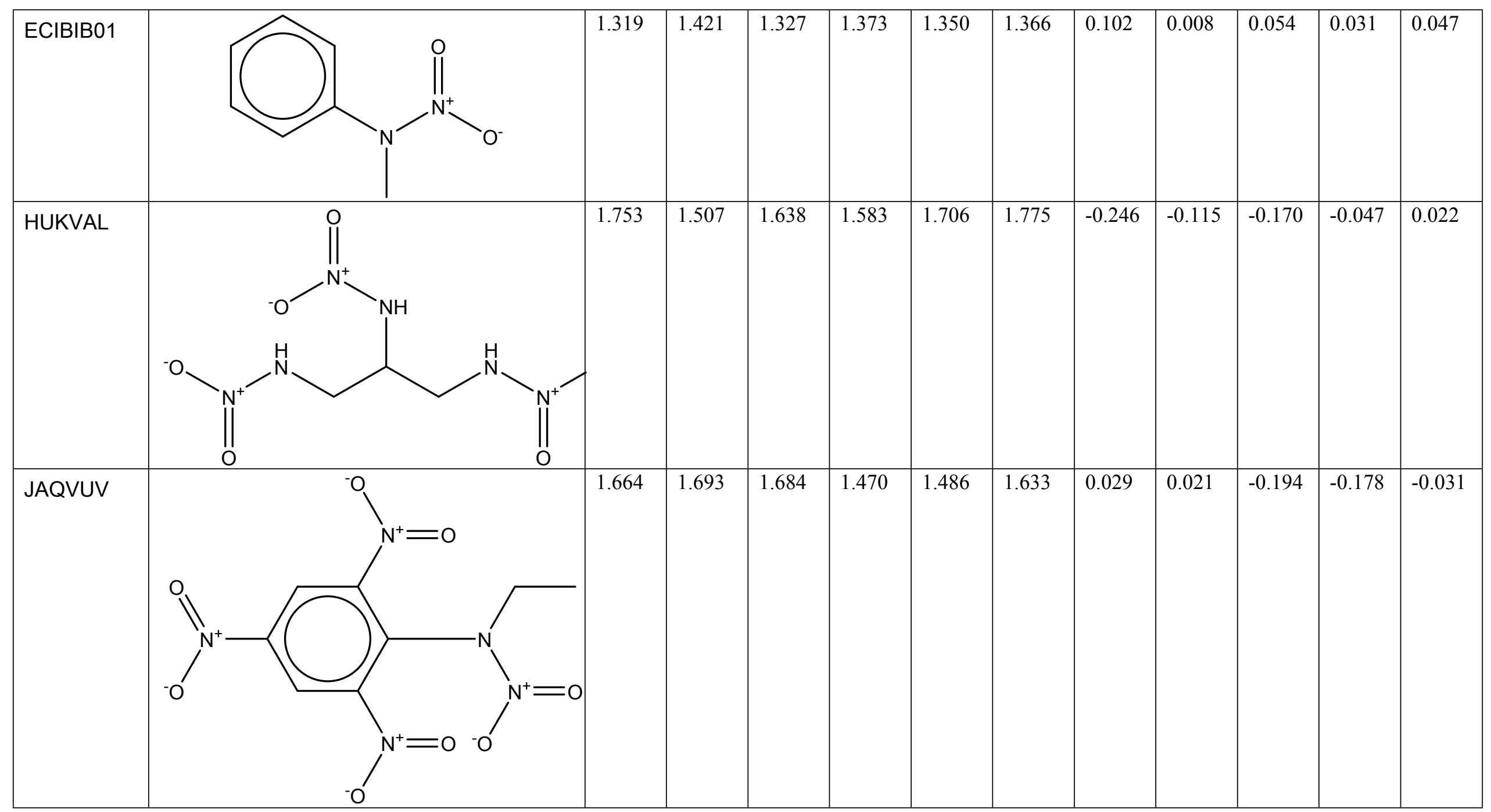




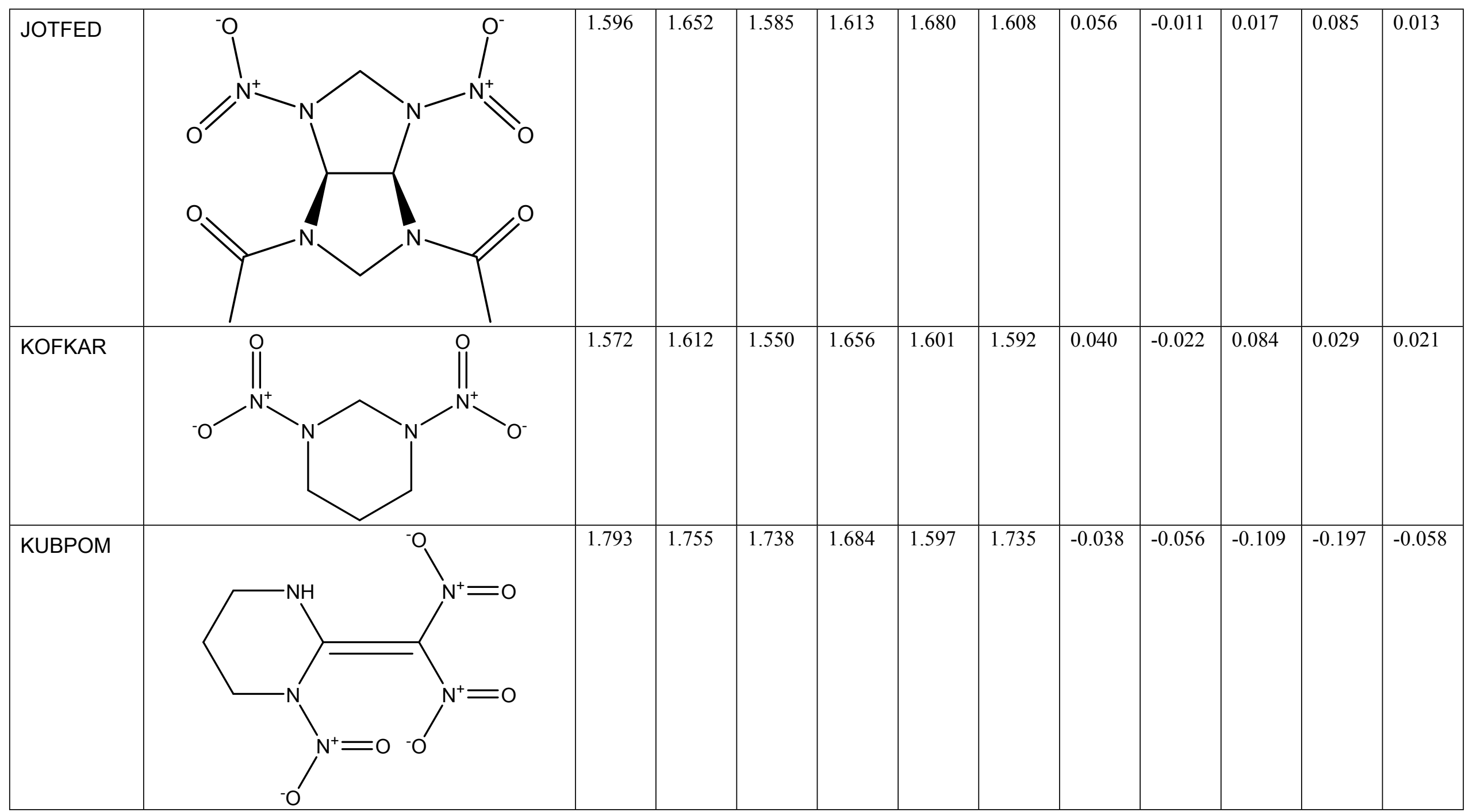




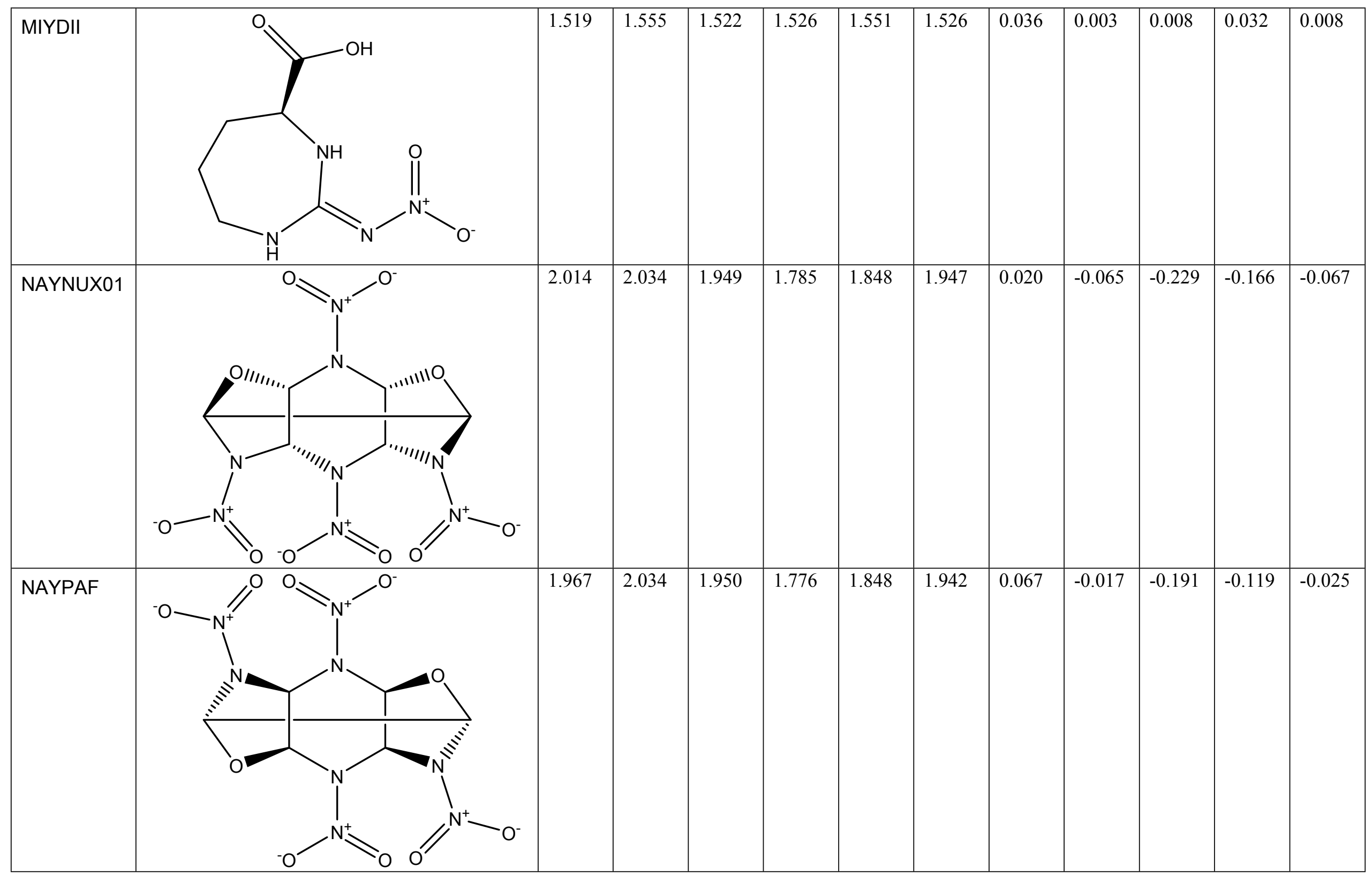




\begin{tabular}{|l|l|l|l|l|l|l|l|l|l|l|l|l|}
\hline UZEWED & 1.718 & 1.897 & 1.782 & 1.724 & 1.596 & 1.793 & 0.179 & 0.064 & 0.006 & 0.122 & 0.075 \\
\hline UZEWIH & & &
\end{tabular}




\begin{tabular}{|c|c|c|c|c|c|c|c|c|c|c|c|c|}
\hline YIQZAA & $\mathrm{O}^{-}$ & 1.383 & 1.369 & 1.379 & 1.400 & 1.411 & 1.401 & -0.014 & -0.004 & 0.017 & 0.028 & 0.018 \\
\hline $\begin{array}{l}\text { CASBOQ0 } \\
1\end{array}$ & 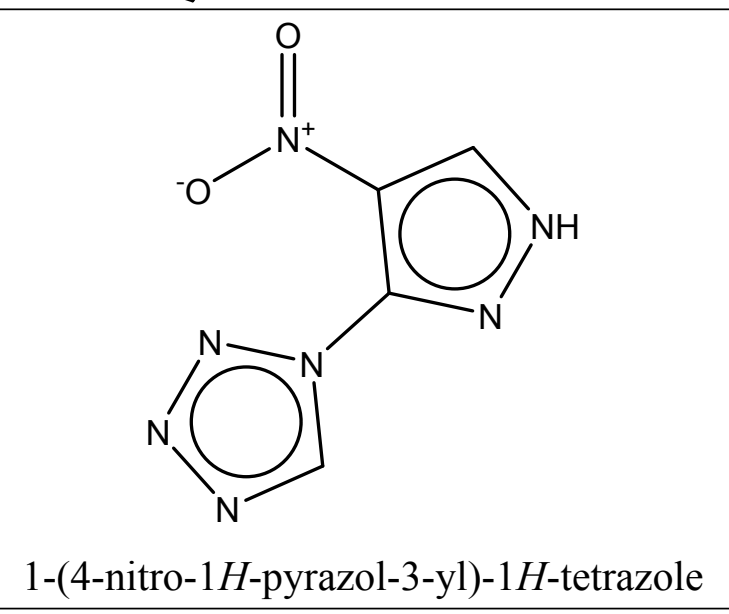 & 1.769 & 1.704 & 1.703 & $\begin{array}{l}1.747 \\
\end{array}$ & 1.767 & 1.643 & -0.065 & -0.066 & -0.022 & -0.002 & -0.127 \\
\hline COGGAI & $\overbrace{}^{N}$ & 1.819 & 1.974 & 1.767 & 1.666 & 1.764 & 1.782 & 0.155 & -0.052 & -0.153 & -0.055 & -0.037 \\
\hline
\end{tabular}




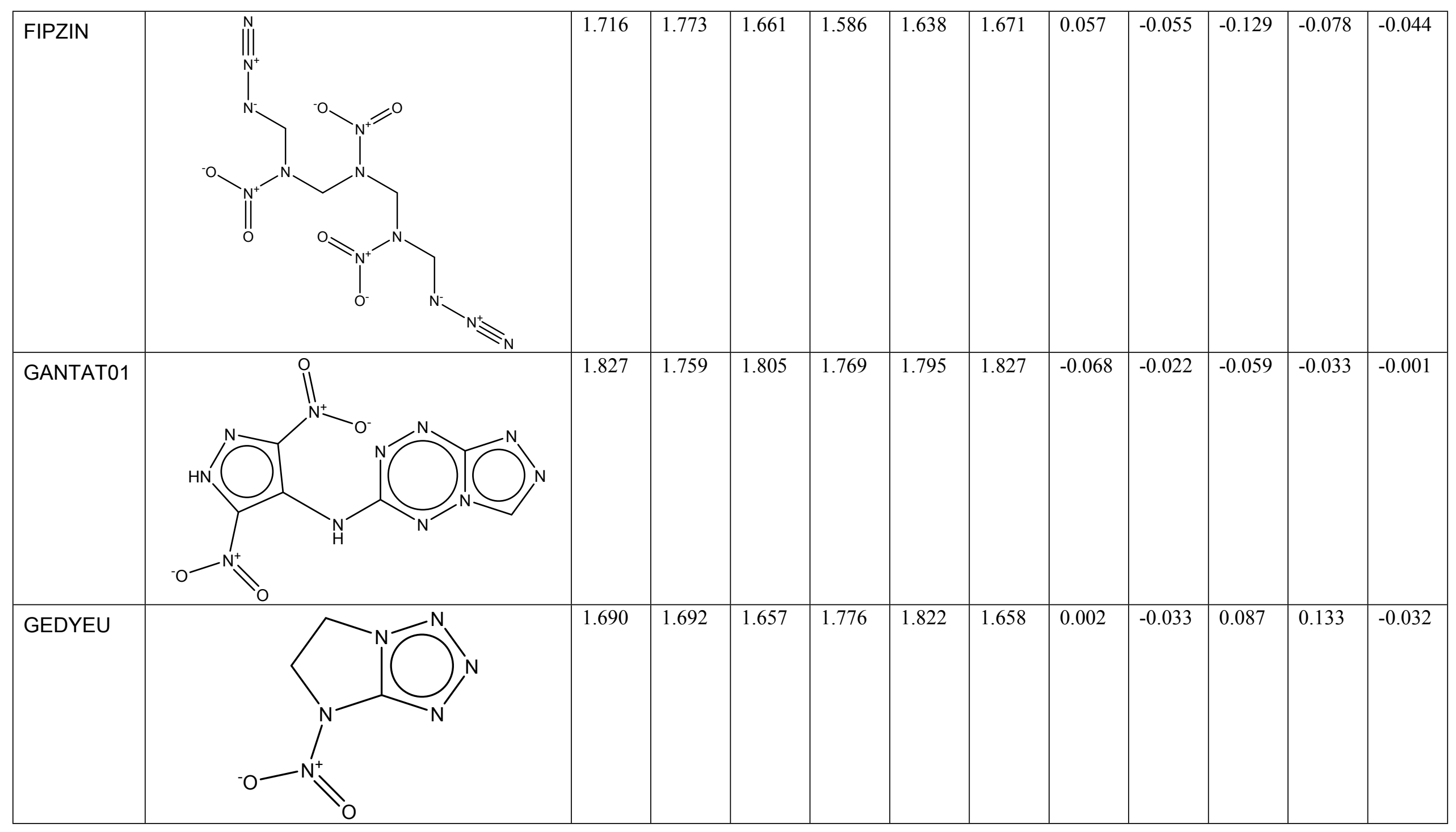




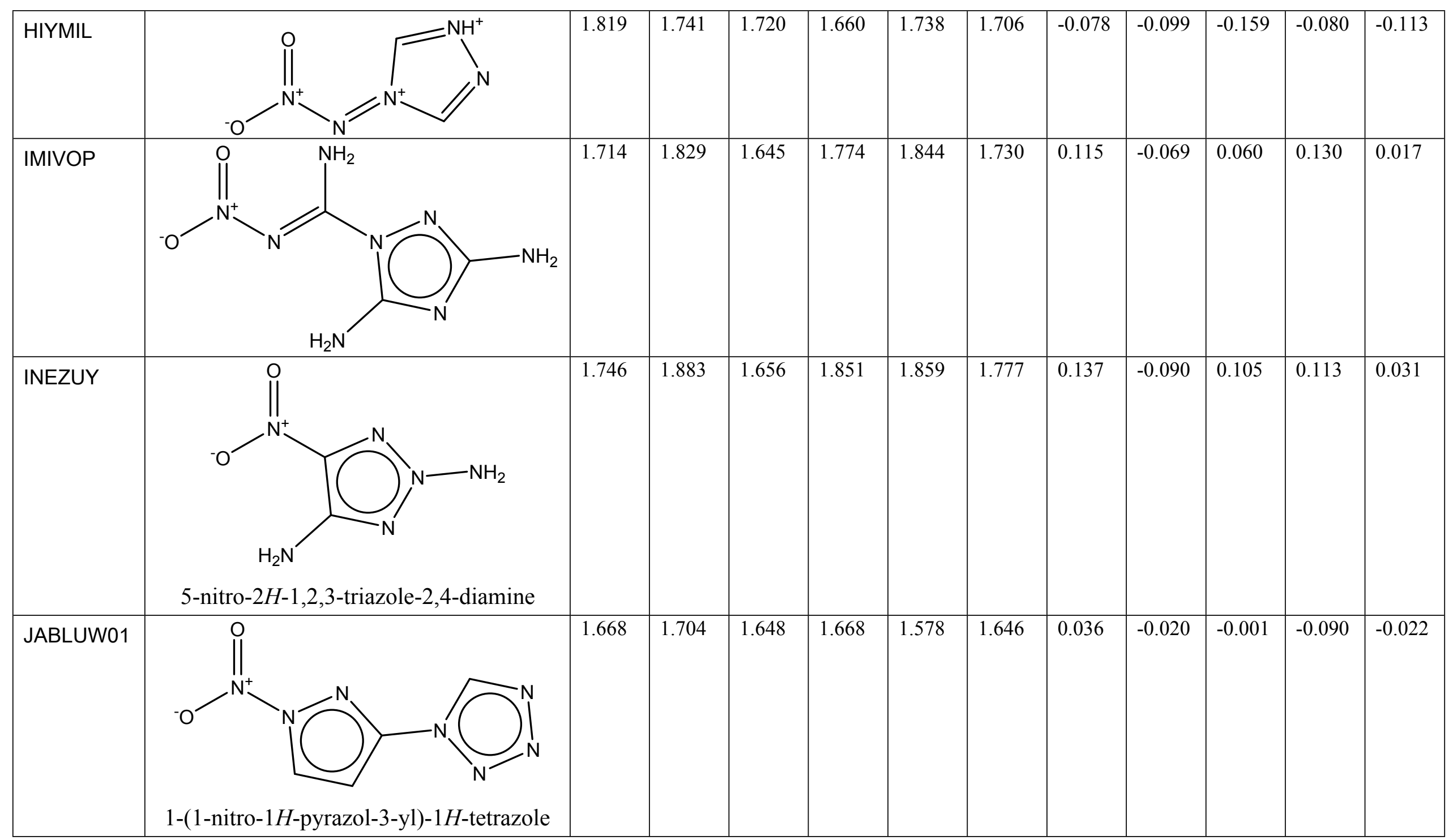




\begin{tabular}{|c|c|c|c|c|c|c|c|c|c|c|c|c|}
\hline JABMAD01 & 1-(1-nitro-1H-pyrazol-4-yl)-1H-tetrazole & 1.728 & 1.704 & 1.650 & 1.731 & 1.730 & 1.638 & -0.024 & -0.079 & 0.002 & . & -0.090 \\
\hline JIPSAF & & 1.794 & 1.756 & 1.782 & 1.757 & 1.767 & 1.864 & -0.038 & -0.013 & -0.038 & -0.027 & 0.070 \\
\hline JOWWIB & 3-nitro-1H-1,2,4-triazol-5-amine & 1.819 & 1.907 & 1.689 & 1.755 & 1.863 & 1.811 & 0.088 & -0.129 & -0.064 & 0.044 & -0.008 \\
\hline MINHAV & $\mathrm{N}_{1}$ & 1.742 & 1.754 & 1.740 & 1.729 & 1.777 & 1.861 & 0.012 & -0.002 & -0.013 & 0.035 & 0.119 \\
\hline
\end{tabular}




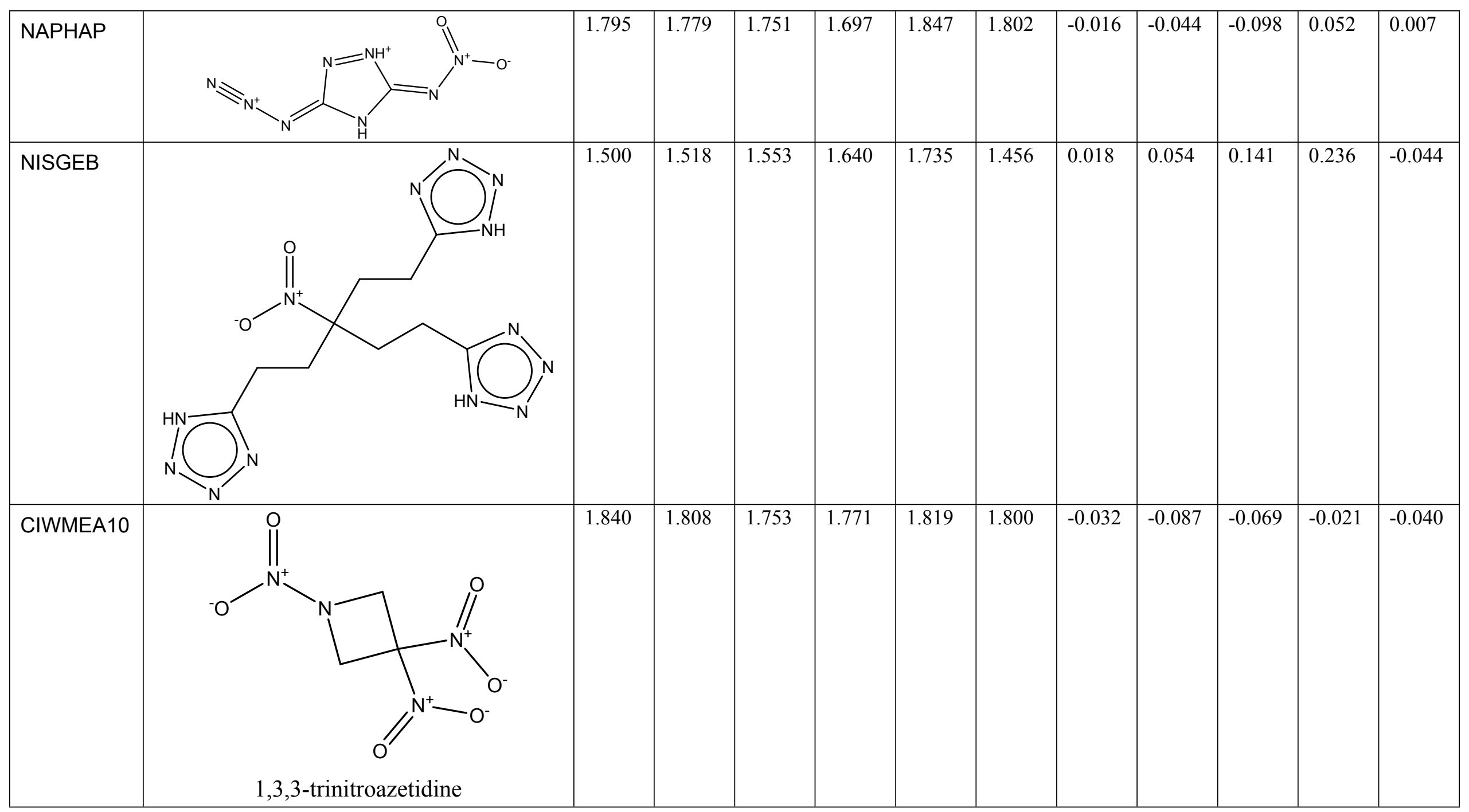




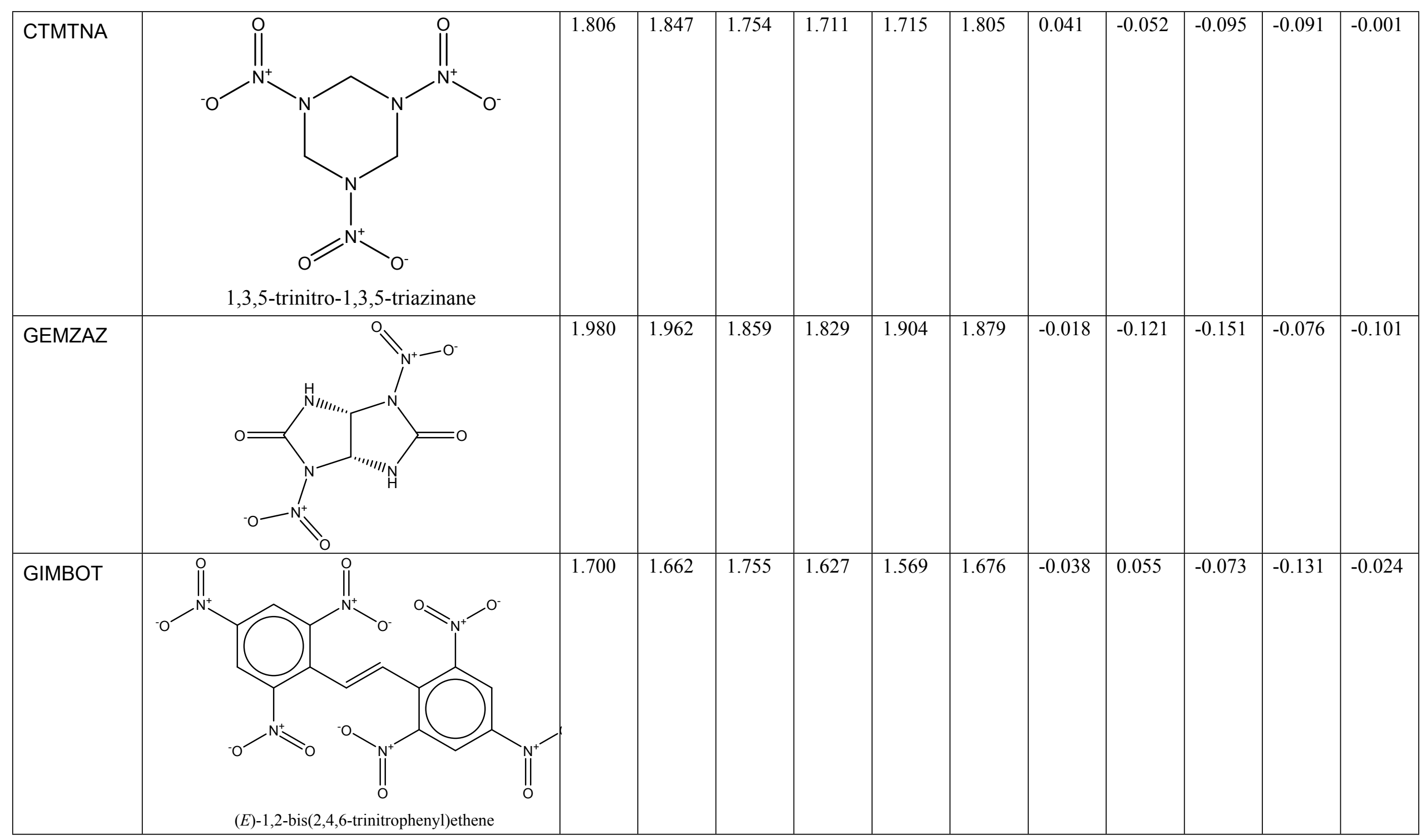




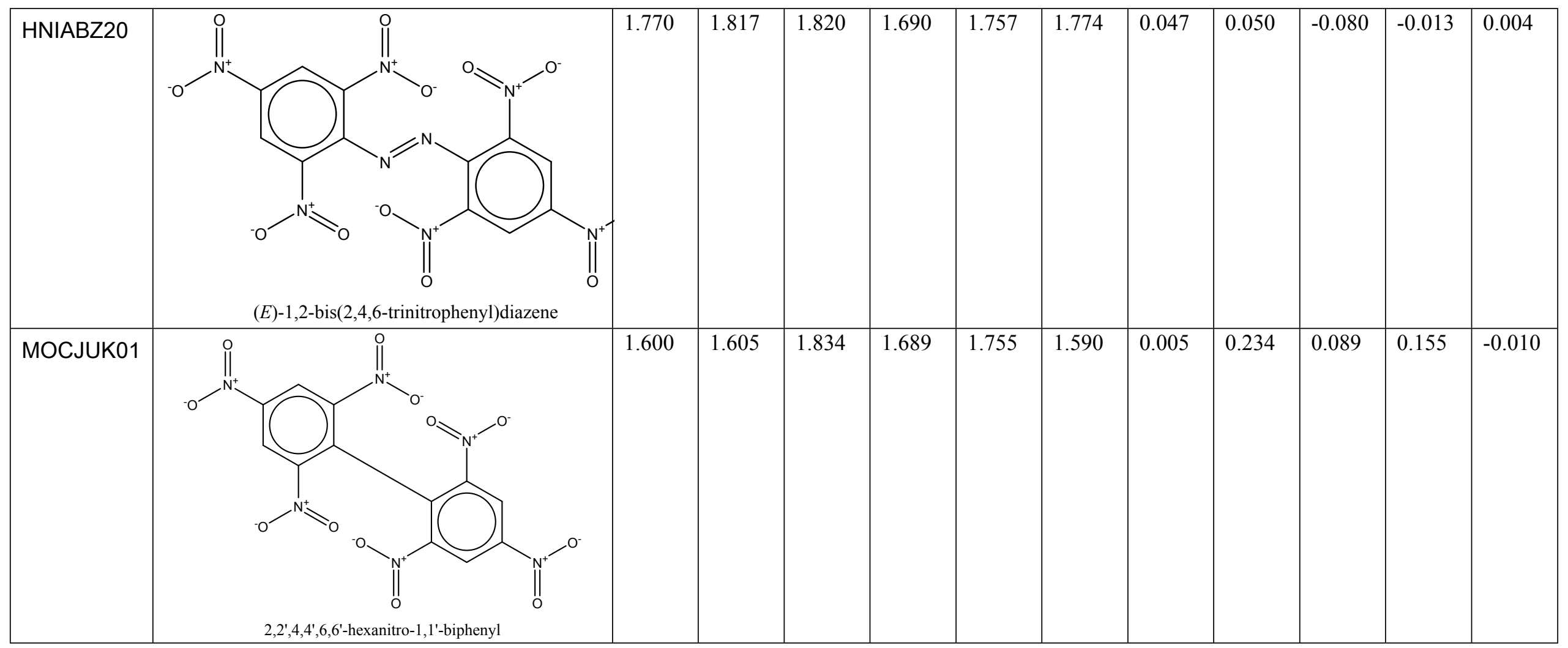




\begin{tabular}{|c|c|c|c|c|c|c|c|c|c|c|c|c|}
\hline MTNANL & 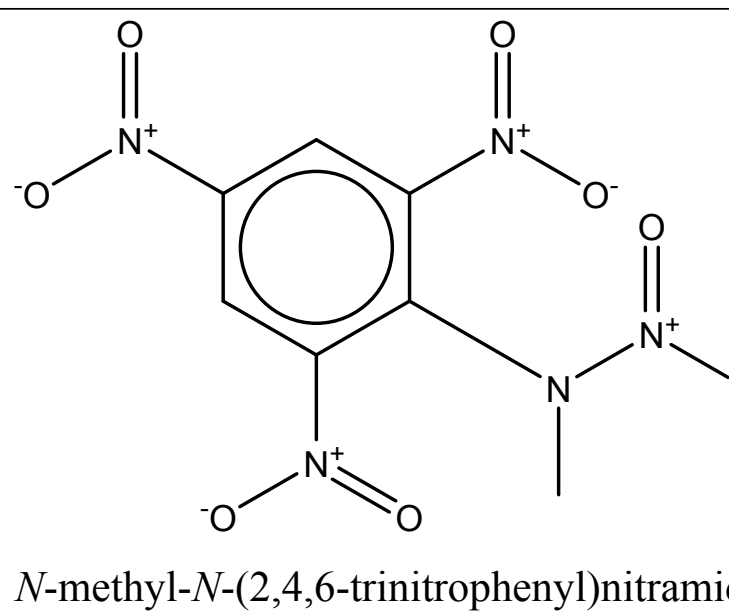 & 1.730 & 1.762 & 1.754 & 1.549 & 1.601 & 1.709 & 0.032 & 0.024 & -0.181 & 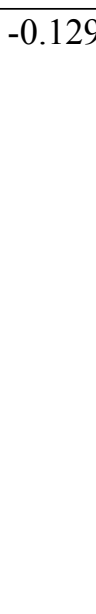 & -0.021 \\
\hline $\begin{array}{l}\text { MUGNUXO } \\
1\end{array}$ & & 1.987 & 1.855 & 1.901 & 1.772 & 1.824 & 1.858 & -0.132 & -0.086 & -0.215 & -0.163 & -0.129 \\
\hline
\end{tabular}




\begin{tabular}{|c|c|c|c|c|c|c|c|c|c|c|c|c|}
\hline OCHTET18 & (1,3,5,7-tetranitro-1,3,5,7-tetrazocane & 1.903 & 1.847 & 1.790 & 1.711 & 1.715 & 1.801 & \begin{tabular}{l|l|l|}
-0.056 \\
\end{tabular} & -0.113 & -0.192 & . & $\begin{array}{r}-0.102 \\
\\
\end{array}$ \\
\hline PICRAC17 & (licic acid & 1.763 & 1.780 & 1.793 & 1.725 & 1.767 & 1.802 & 0.017 & 0.030 & -0.038 & 0.004 & 0.039 \\
\hline
\end{tabular}




\begin{tabular}{|c|c|c|c|c|c|c|c|c|c|c|c|c|}
\hline $\begin{array}{l}\text { PUBMUU2 } \\
0\end{array}$ & $\mathbb{N}_{\mathrm{N}^{+}}^{\mathrm{O}} \mathrm{O}^{-0} \backslash_{\mathrm{N}^{+}}=0$ & 2.040 & 2.009 & 1.990 & 1.753 & 1.852 & 1.971 & -0.031 & -0.050 & -0.287 & -0.188 & -0.069 \\
\hline QOYJOD06 & 5-nitro-2,4-dihydro-3H-1,2,4-triazol-3-ol & 1.910 & 1.911 & 1.752 & 1.753 & 1.869 & 1.864 & 0.001 & -0.158 & -0.157 & -0.041 & -0.046 \\
\hline SEDTUQ03 & 2,2-dinitroethene-1,1-diamine & 1.893 & 2.057 & 1.750 & 1.662 & 1.791 & 1.855 & 0.164 & -0.143 & -0.231 & -0.102 & -0.038 \\
\hline
\end{tabular}




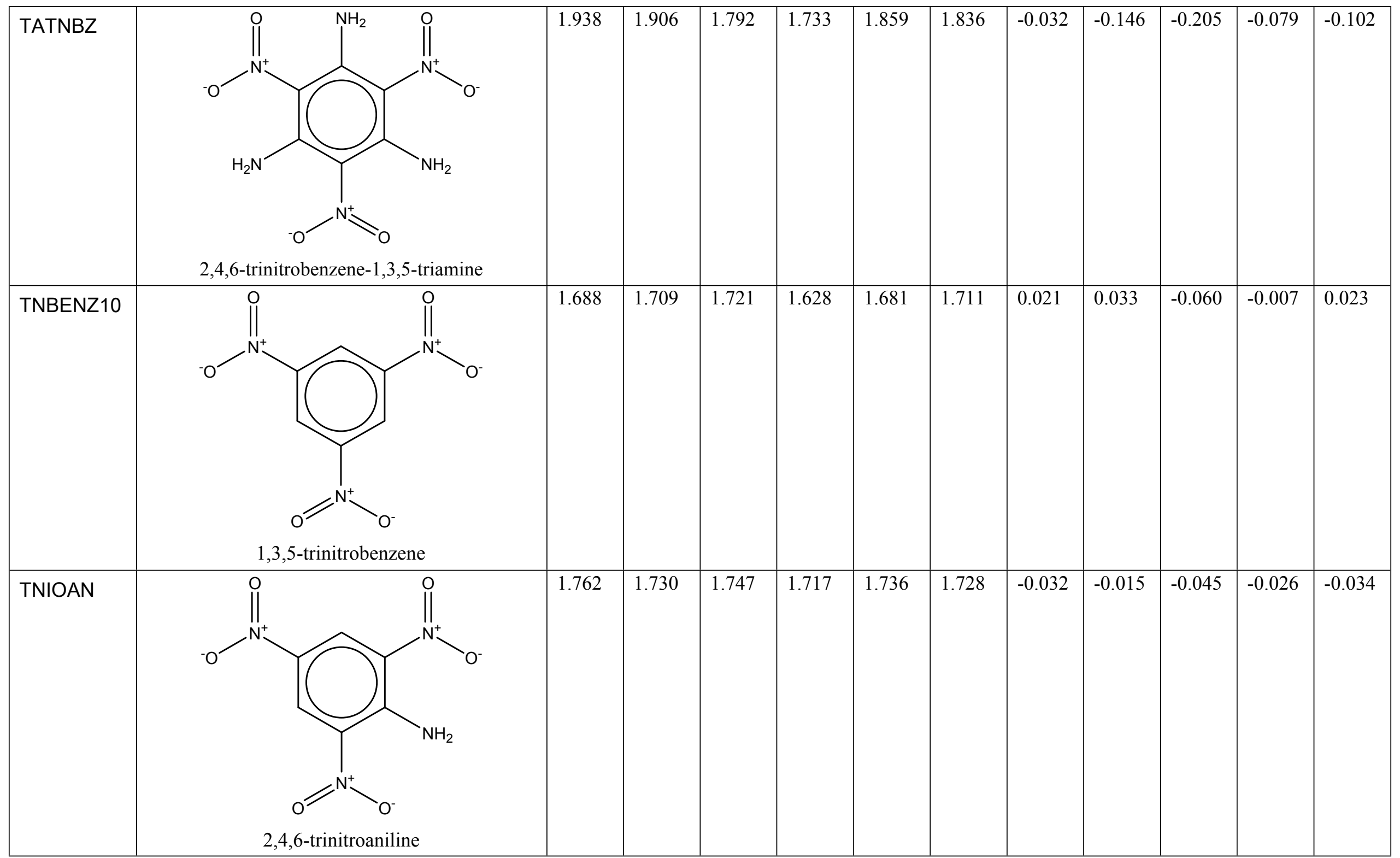




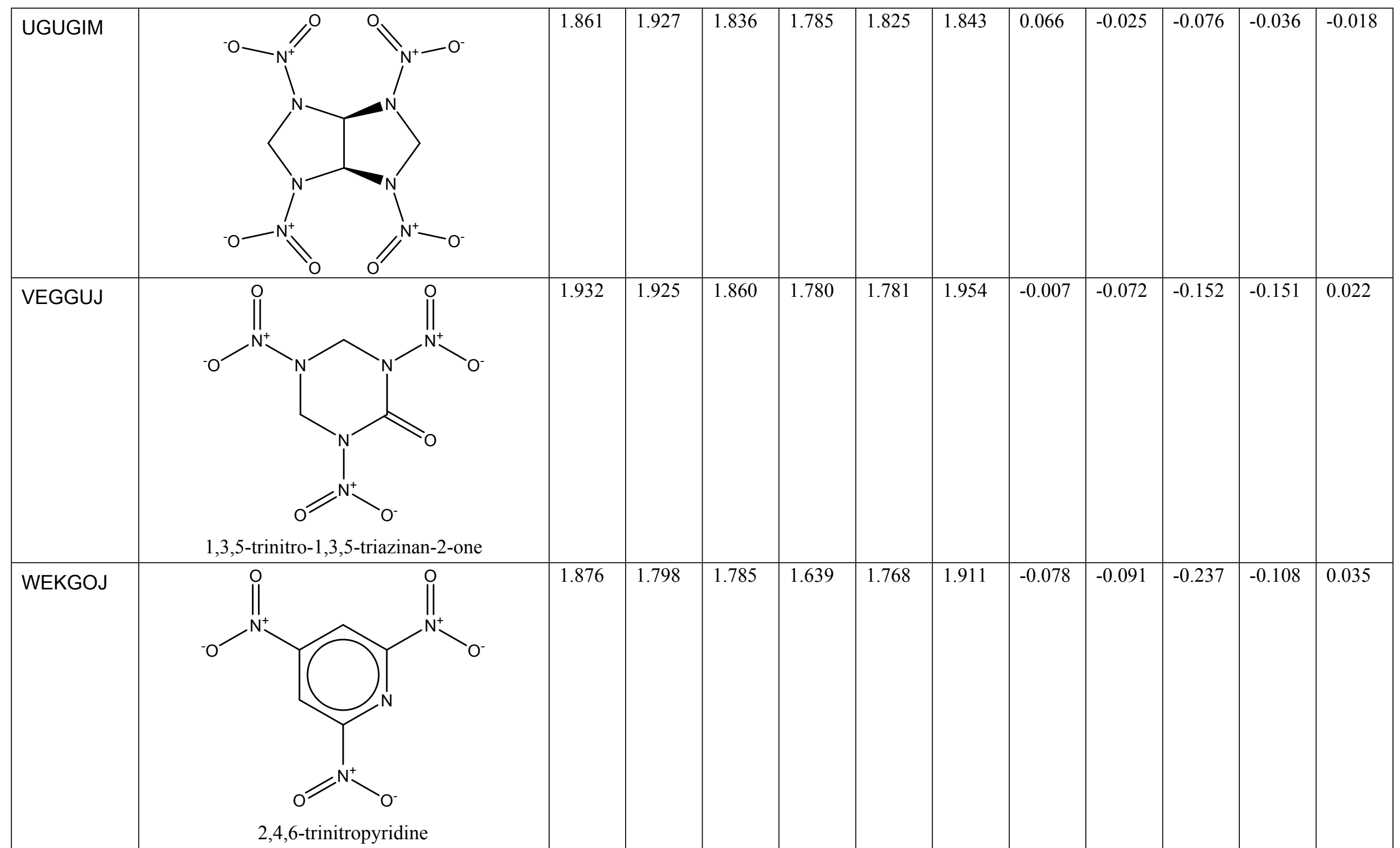




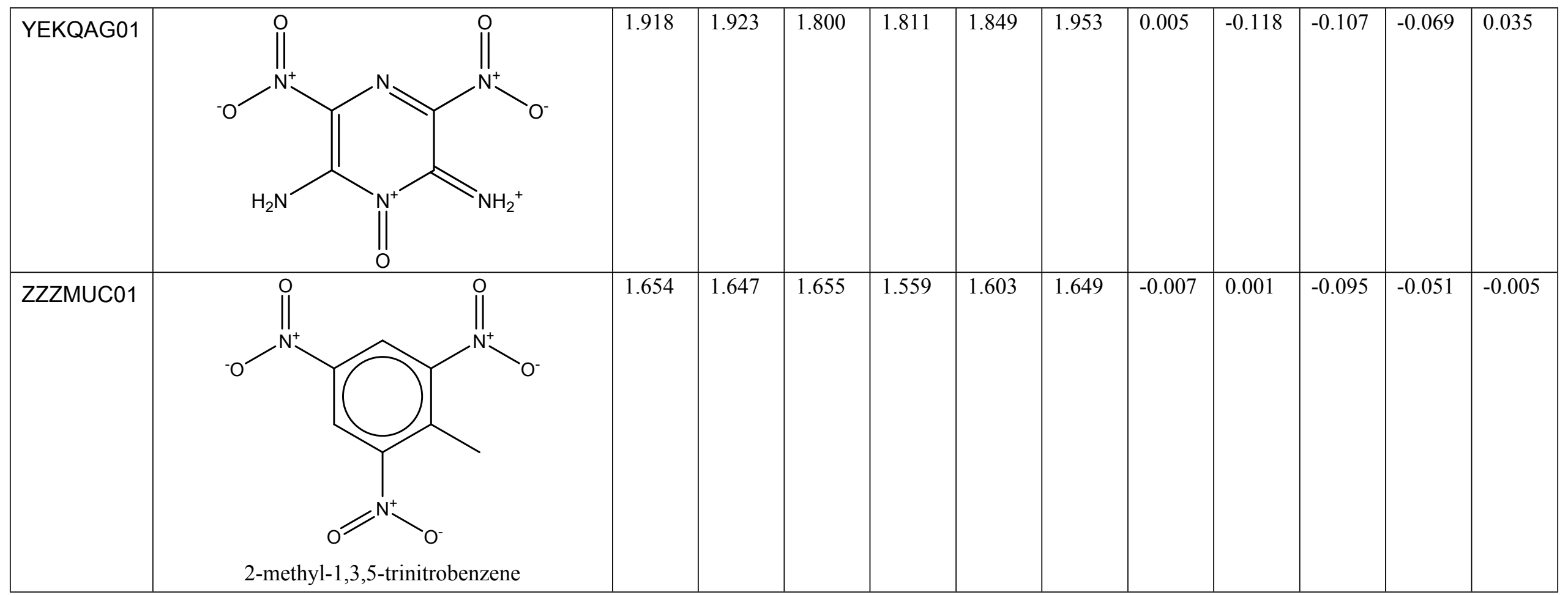

